\title{
SHORT COMMUNICATION Numerical Evaluation of the Resistivity of Polycrystalline Metal Films with the Mayadas-Shatzkes Model
}

\author{
E. E. MOLA and J. M. HERAS \\ Instituto de Investigaciones Fisicoquimicas Teóricas y Aplicadas, Division Fisicoquimica de \\ Superficies, Calle 47 y 115, La Plata, R. Argentina
}

(Received June 26, 1973)

\begin{abstract}
Numerical Tables are given in order to allow a direct comparison of the electrical resistivity model for polycrystalline films proposed by Mayadas-Shatzkes with experimental data.

The tables have been calculated extending the Gauss method for one variable to many variables.
\end{abstract}

\section{INTRODUCTION}

In the past years, it has been a common practice to analyze electrical resistivity data of polycrystalline metal films ${ }^{1}$ by means of the Fuchs-Sondheimer model $^{2}$ (F-S model) for the size effects. However, the granular structures of evaporated films impose a restriction about which the F-S model says nothing. In order to take this into account, Mayadas and Shatzkes ${ }^{3}$ developed the model (M-S model) given briefly later on.

The purpose of this communication is to present numerical tables. Model possibilities and applications have been discussed elsewhere ${ }^{4}$.

\section{MAYADAS-SHATZKES MODEL}

Basically, to the two electron scattering mechanisms of the well known F-S model, M-S add a third one due to the presence of crystallite boundaries.

For the evaluation of the overall film resistivity $\rho_{\mathrm{f}}, \mathrm{M}-\mathrm{S}$ assumed that crystallite boundaries can be represented by a Gaussian distribution of partially electron reflecting planes randomly spaced with an average distance $a$ and a standard deviation $s$.

Based on the available experimental data, which shows that thin evaporated films have a column-like structure with crystallites towering from the substrate to the surface, they assumed that (i) only those planes perpendicular to the applied electric field cause the extra electron scattering; (ii) the average interplanar distance $a$ is equivalent to the average crystallite diameter D as obtained by electron microscopy. In order to fit the model to experimental data, the electron reflecting power of the potential barrier between crystallites is interpreted by a phenomenological parameter $r$.

The model can be summarized by the two following equations, rearranged according to the heading assumptions to make the mathematical problem and nume rical evaluation more tractable.

\subsection{Crystallite Diameter D is a Thickness Independent Constant.}

The ratio between film resistivity $\rho_{\mathrm{f}}$ and the resistivity of a polycrystalline film infinitely thick, $\rho_{\mathrm{g}}$ is given by:

$$
\frac{\rho_{\mathrm{f}}}{\rho_{\mathrm{g}}}=\left[1-\frac{\mathrm{A}}{\mathrm{f}_{(\alpha)}}\right]^{-1}
$$

in which:

$$
\begin{gathered}
\mathrm{A}=\frac{6}{\mathrm{k}_{0} \pi}(1-\mathrm{p}) \int_{0}^{\pi / 2} \mathrm{~d} \varphi \int_{1}^{\infty} \frac{\operatorname{Cos}^{2} \varphi}{\mathrm{H}^{2}(\mathrm{t}, \varphi)}\left(\frac{1}{\mathrm{t}^{3}}-\frac{1}{\mathrm{t}^{5}}\right) \\
\times \frac{1-\mathrm{e}^{-\mathrm{k}_{0} \mathrm{tH}(\mathrm{t}, \varphi)}}{1-\mathrm{pe}^{-\mathrm{k}_{0} \mathrm{tH}(\mathrm{t}, \varphi)}}
\end{gathered}
$$

with:

$$
\mathrm{H}(\mathrm{t}, \varphi)=1+\alpha /\left(1-\frac{1}{\mathrm{t}^{2}}\right)^{1 / 2} \operatorname{Cos} \varphi
$$

$\mathrm{k}_{0}=\mathrm{d} / 1_{0} ; \mathrm{d}=$ film thickness; $1_{0}=$ mean free path of the electrons; $p=$ specularity parameter. 
TABLE I

Numerical data according to Eq. (1). $\mathrm{p}=$ specularity parameter; $\mathrm{k}_{0}=d / \mathrm{l}_{0} ; d=$ film thickness; $\mathbf{1}_{0}=$ electron mean free path; $\alpha=\left(l_{0} / \mathrm{D}\right) \mathrm{r} / 1-\mathrm{r} ; \mathrm{D}=$ crystalline diameter; $r=$ reflection coefficient.

\begin{tabular}{|c|c|c|c|c|c|c|c|c|}
\hline \multirow[b]{2}{*}{$k_{0}$} & \multicolumn{4}{|c|}{$p=0$} & \multicolumn{4}{|c|}{$p=0.5$} \\
\hline & $\alpha=0$ & $\alpha=0.5$ & $\alpha=1.0$ & $\alpha=2.0$ & $\alpha=0$ & $\alpha=0.5$ & $\alpha=1.0$ & $\alpha=2.0$ \\
\hline 0.01 & 23.1528 & 17.6710 & 13.3803 & 9.4128 & 11.9282 & $7 \circ 9603$ & 6.2672 & 4.6383 \\
\hline 0.02 & 15.5631 & 10.1334 & 7.8643 & 5.7071 & 7.1911 & 4.9550 & 3.9889 & 3.0523 \\
\hline 0.03 & 11.2719 & 7.4992 & 5.8937 & 4.3444 & 5.4583 & 3.8384 & 3.1370 & 2.4567 \\
\hline 0.04 & 9.0703 & 6.1173 & 4.8468 & 3.6133 & 4.5336 & 3.2394 & 2.6794 & 2.1371 \\
\hline 0.05 & 7.7033 & 5.2498 & 4.1852 & 3.1516 & 3.9439 & 2.8599 & 2.3396 & 1.9351 \\
\hline 0.06 & 6.7707 & 4.6465 & 3.7245 & 2.3302 & 3.5415 & 2.5954 & 2.1870 & 1.7948 \\
\hline 0.07 & 6.0821 & 4.2010 & 3.3843 & 2.5934 & 3.2403 & 2.4000 & 2.0389 & 1.6916 \\
\hline 0.08 & 5.5512 & 3.8567 & 3.1216 & 2.4109 & 3,0073 & 2.2489 & 1,9239 & 1.6122 \\
\hline 0.09 & 5.1271 & 3.5814 & 2.9118 & 2. 2655 & 2.8203 & 2.1282 & 1.8321 & 1,5490 \\
\hline 0.10 & 4.7304 & 3.3565 & 2.7405 & 2.1469 & 2.6632 & 2.0295 & 1.7572 & 1.4976 \\
\hline 0.20 & 3.0962 & 2.2682 & 109154 & 1. 5813 & 1.9251 & 1.5523 & 1.3934 & 1.2545 \\
\hline 0.30 & 2.4659 & 108649 & 1.6131 & 1.3797 & 1.6464 & 1.3775 & 1.2684 & 1.1688 \\
\hline 0.40 & 2.1284 & 1.6515 & 1.4554 & 1.2775 & 1.4975 & 1.2855 & 1.2012 & 1.1252 \\
\hline 0.50 & 1.9161 & 1.5132 & 1.3590 & $1 \cdot 2167$ & 1,4041 & 1.2288 & 1.1601 & 1.0991 \\
\hline 0.60 & 1.7696 & 104292 & 1.2945 & 1.176 & 1.3399 & 1,1903 & 1,1326 & 1.0818 \\
\hline 0.70 & 1.6621 & 1.3643 & 1.2485 & 1.1489 & 1.2930 & 1.1626 & 1.1129 & 1.0696 \\
\hline 0.80 & 1.5799 & 1.3154 & 1.2143 & 1. 1284 & 1.2572 & 1.1417 & 1.0981 & 1.0604 \\
\hline 0.90 & 1.5149 & 1.2773 & 1.1380 & 1.1127 & 1.2289 & 1.1254 & 1.0367 & 1.0534 \\
\hline 1.00 & 104623 & 1.2470 & 1.1671 & 1.1004 & 1.2061 & 1.1123 & 1.0776 & 1,0478 \\
\hline 2.00 & 1.2203 & 1.1146 & 1.0780 & 1.0479 & 1.1014 & 1.0542 & 1.0376 & 1.0234 \\
\hline 3.00 & 1.1414 & 1.0737 & 1.0507 & 1.0314 & 1.0663 & 1.0356 & 1.0247 & 1.0155 \\
\hline 4000 & 1.1032 & 100543 & 1.0376 & 1.0234 & 100491 & 1.0264 & 1.0184 & 1.0116 \\
\hline 5.00 & 1.0810 & 1.0430 & 1.0298 & 1.0186 & 1.0389 & 1.0210 & 1.0147 & 1.0092 \\
\hline 6.00 & 1.0666 & 1.0356 & 1.0247 & 1.0155 & 1.0322 & 1.0175 & 1.0122 & 1.0077 \\
\hline 7.00 & 1.0566 & 1.0303 & 1.0211 & 1.0132 & 1.0275 & 1.0149 & 1.0104 & 1.0066 \\
\hline 3.00 & 1.0492 & 1.0264 & 1.0184 & 1.0116 & 1.0240 & 1.0130 & 1.0091 & 1.0057 \\
\hline 9.00 & 1.0435 & 1.0234 & 1.0164 & 1.0102 & 1.0213 & 1.0116 & 1.0081 & 1.0051 \\
\hline 10.00 & 1.0390 & 1.0210 & 1.0147 & 1.0092 & 1.0191 & 1.0104 & 1.0073 & 1.0046 \\
\hline
\end{tabular}

$$
\alpha=\left(1_{0} / \mathrm{D}\right) \mathrm{r} / 1-\mathbf{r}
$$

and

$$
f_{(\alpha)}=1-(3 / 2) \alpha+3 \alpha^{2}-3 \alpha^{3} \ln (1+1 / \alpha)
$$

When $\alpha=0$, Eq. (1) reduces to the F-S equation. Neverthelss the curve shapes are identical within the experimental error.

\subsection{Crystallite Diameter D is Equal to Film Thickness $d$.}

Then, the ratio between film resistivity $\rho_{\mathrm{f}}$ and the resistivity of a single crystal film infinitely thick, $\rho_{0}$, is:

$$
\frac{\rho_{\mathrm{f}}}{\rho_{0}}=\left[\mathrm{f}_{(\alpha)}-\mathrm{A}\right]^{-1}
$$

in which $A, f_{(\alpha)}$ and $\alpha$ have the same meaning as in eq. (1). When $\mathrm{k}_{0}>10$ or $\mathrm{r} \rightarrow 0$, eq. (2) reduces to the F-S equation.

\section{NUMERICAL TABLES}

Unfortunately Eq. (1) and (2) cannot be evaluated analytically. Hence, in order to interpret quantitatively the experimental results it was necessary to 
elaborate numerical tables. These have not been given in the literature yet and were obtained with a IBM-360/50 computer with a program written in PLI. Excessively high computer time is necessary to solve the integrals by conventional methods ${ }^{5}$. This problem was overcome by generalizing Gauss' equation $^{6}$ to any number of variables. This can be done changing the variables in such a way as to transform the original interval for each variable into another one, ranging from 0 to 1 , as required by the Gauss' formula. The integral is then evaluated over $\mathrm{n}^{2}$ points, each one of them given by the roots of the Legendre polynomials of order $\mathrm{n}$ with their respective weight. $^{7}$
The numerical evaluation was carried over $\mathrm{n}^{2}=100$ points to obtain an acceptable approximation. Nevertheless, 25 points are enough to maintain error below $1 \%$ for the integrals, but their place in the function magnifies the overall error.

In Table I the values of $\rho_{\mathrm{f}} / \rho_{\mathrm{g}}$ vs. $\mathrm{k}_{0}$ calculated with Eq. (1) are given under the assumption $\alpha=a$ thickness independent constant. Only two values of the specularity parameter $\mathrm{p}$ are taken into account, because in general the experimental data fit the theoretical values better by postulating $p=0$.

Table II shows the dependence of $\rho_{\mathrm{f}} / \rho_{\mathrm{o}}$ on $\mathrm{k}_{0}$ calculated with Eq. (2) for the same p-values as in Table I, but with $\alpha=\mathrm{f}(\mathrm{d})$, (i.e. $\mathrm{D}=\mathrm{d}$ and $\mathrm{r}=$ thickness

TABLE II

Numerical data according to Eq. (2). p, $\mathrm{k}_{0}$ and $r$ have the same significance as in Table I.

\begin{tabular}{|c|c|c|c|c|c|c|c|c|}
\hline \multirow[b]{2}{*}{$k_{0}$} & \multicolumn{4}{|c|}{$p=0$} & \multicolumn{4}{|c|}{$p=0.5$} \\
\hline & $r=0.1$ & $r=0.22$ & $r=0.42$ & $r=0.62$ & $r=0.1$ & $r=0.22$ & $r=0.42$ & $r=0.62$ \\
\hline 0.01 & 54.3700 & 31.0040 & 139.7323 & 257.4954 & 32.3003 & 57.0046 & 116.2411 & 236.6560 \\
\hline 0.02 & 23.1572 & 41.0842 & 70.3962 & 129.2750 & 17.0153 & 29.0588 & 58.6566 & 118.8614 \\
\hline 0,03 & 19.2322 & 27.7615 & $47 \cdot 2602$ & 36.4922 & 11.7423 & 19.7326 & 39.4419 & 79.5564 \\
\hline 0.04 & $14=7706$ & 21.1111 & 35.7139 & 65.1412 & 9.1070 & 15.0774 & 29.3525 & 59.9413 \\
\hline 0.05 & 12.0376 & 17.1187 & 28.7344 & 52.3278 & 7.5226 & 12.2829 & 24.0975 & 48.1697 \\
\hline 0.06 & 10.2924 & 14.4523 & 24.1581 & 43.7736 & 6.4629 & 10.4166 & 20.2553 & 40,3109 \\
\hline 0.07 & 900092 & 12.5500 & 20.8589 & 37.6735 & 5.7056 & 9.0853 & 17.5153 & 31.7068 \\
\hline 0.03 & 8.0442 & 11.1224 & 1303841 & 33.0978 & 5.1364 & 8.0863 & 15.4599 & 30.5030 \\
\hline 0.03 & 7.2906 & 10.0093 & 16.4563 & 29.5337 & 4.6919 & 7.3078 & 13.8538 & 27.2287 \\
\hline 0.10 & 6.6370 & 9.1207 & 14.9164 & 26.6370 & 4.3361 & 6.6857 & 12.5799 & 24.6133 \\
\hline 0.20 & 3.9367 & 5.1055 & 709302 & $13 \cdot 8682$ & 2. 7176 & 3.3778 & 6,8190 & 12.8364 \\
\hline 0.30 & 2.9947 & 3.7570 & 5.6658 & 9.5952 & 2.1657 & 2.9360 & 4.8966 & 8.9104 \\
\hline 0.00 & 2.5127 & 3.0775 & 4.5070 & 7. 4578 & 1.8846 & 2.4619 & 3.9338 & 6.9463 \\
\hline 0.50 & 2.2130 & 2.6670 & 3.3107 & 6.1749 & 1.7133 & 2.1758 & 3.3551 & 5.7673 \\
\hline 0.60 & 2.0106 & 2.3917 & 3.3459 & $5 \cdot 3194$ & 1.5978 & 1.9840 & 2.9687 & 4.9810 \\
\hline 0.70 & 1.8744 & 2.1940 & 3.0134 & 4.7079 & 1.5144 & 1.8464 & 2.6922 & 4.4189 \\
\hline 0.80 & 1.7652 & 2.0451 & 2.7638 & 4.2492 & 1.4513 & 1.7427 & 2.4844 & 3.9971 \\
\hline 0.90 & 1.6796 & 109238 & 2. 5694 & 3.3922 & 1.4020 & 106617 & 2.3226 & 3.6688 \\
\hline 1.00 & 1.6107 & 1.8356 & 2.4137 & 3.6064 & 1.3622 & 1.5967 & 2.1928 & 3,4060 \\
\hline 20.00 & .1.2972 & 1.4134 & 1.7108 & 2.3174 & 1.1811 & 1.3011 & 1.6053 & 2,2194 \\
\hline 3.00 & 1.1936 & 1.2730 & 1. 4753 & 1.8852 & 1.1201 & 1.2014 & 1.4071 & 1.8207 \\
\hline 4.00 & 1.1430 & 1.2034 & 1. 3574 & 1.6679 & 1.0398 & 1.1513 & 1.3071 & 1.6199 \\
\hline 5000 & 1.1132 & 1.1620 & 1.2864 & 1.5370 & 1.0716 & 1.1211 & 1.2467 & 1.4988 \\
\hline 6.00 & 1.0936 & 1.1347 & 1.2391 & 1.4492 & 1.0596 & 1.1010 & 1.2063 & 1.4175 \\
\hline 7.00 & 1.0798 & 1.1152 & 1.2052 & 1. 3364 & 1.0510 & 1.0366 & 1.1773 & 1.3592 \\
\hline 8.00 & 1.0696 & 1.1006 & 1.1798 & 1.3390 & 1.0446 & 1.0759 & 1.1555 & 1.3154 \\
\hline 9.00 & 1.0616 & 1.0894 & 1.1600 & 1.3021 & 1.0396 & 1.0675 & 1.1335 & 1.2811 \\
\hline 10.00 & 1.0554 & 1.0303 & 1.1441 & 1.2725 & 1.0356 & 1.0607 & 1.1243 & 1.2536 \\
\hline
\end{tabular}


independent constant). There are more curves given with different $r$, than with different $\alpha$ because actually the available data fit the former more closely.

In Table I the column corresponding to $\alpha=0$ is actually the numerical data of the F-S equation. A column with $r=0$ in Table II would be identical. These values are given in order to estimate the error of the method. For this purpose they should be compared with the data obtained by Soffer ${ }^{8}$ with Simpson's rule using $2^{13}$ subdivisions. The error is less than $0.1 \%$ up to $\mathrm{k}_{0}=0.05$. However, the first value for $\mathrm{k}_{0}=0.01$ has an error of $6 \%$.

The evaluation method is thus justified because the computation time was reduced to 30 seconds per column.

\section{ACKNOWLEDGMENT}

The authors are indebted to the Director of the Institute, Prof. Dr. H. J. Schumacher for his interest, and to Dr. J.
Gordon, Director of the Calculus Center (CESPI) for many useful discussions on mathematical problems. One of us (E. E. M.) is also grateful to the CONICET (R. Argentina) for a scholarship.

\section{REFERENCES}

1. See a) J. Le Bas, Compt. Rend. (Paris) 268 B 262 (1969). b) G. Wedler, M. Fouad, Z. physik. Chem. N. F. 401 (1963).

2. a) K. Fuchs, Proc. Cambridge Phil. Soc. 34100 (1938). b) E. H. Sondheimer, Adv. Phys., 1 (1952).

3. A. F. Mayadas and M. Shatzkes, Phys. Rev. B 11382 (1970).

4 E. E. Mola, J. Borrajo and J. M. Heras, Surface Sc. 34561 (1973).

5. V. I. Krylov, Approximate Calculation of Integrals. (Macmillan, New York, 1962.)

6. J. Singen, Elements of Numerical Analysis, (Academic Press, New York, London, 1964.)

7. J. Gordon, Universidad Nacional de La Plata, CESPI, calle 50 y 115 . La Plata, R. Argentina. Private communication. 8. S. Soffer, J. Appl. Phys., 363947 (1965). 

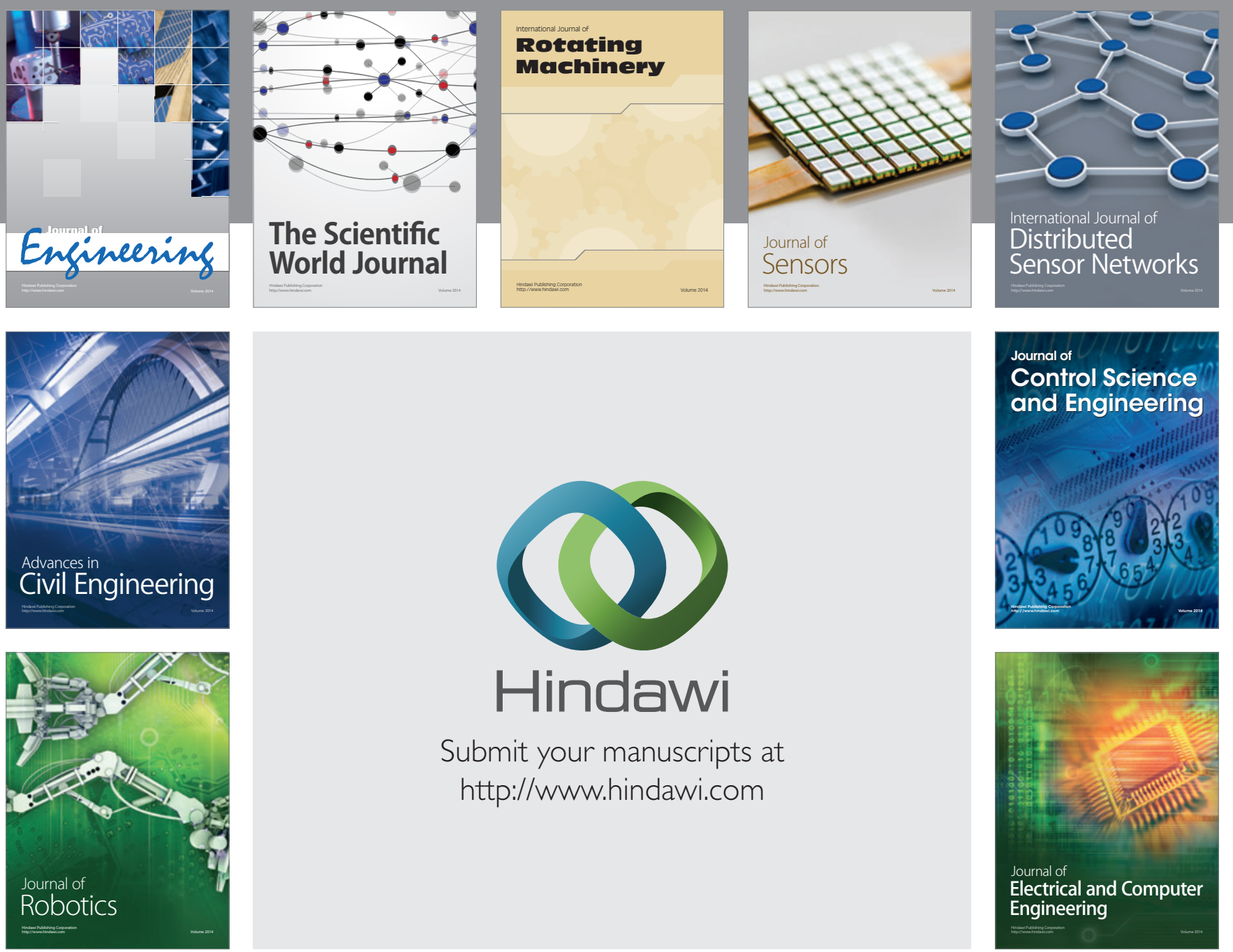

Submit your manuscripts at

http://www.hindawi.com
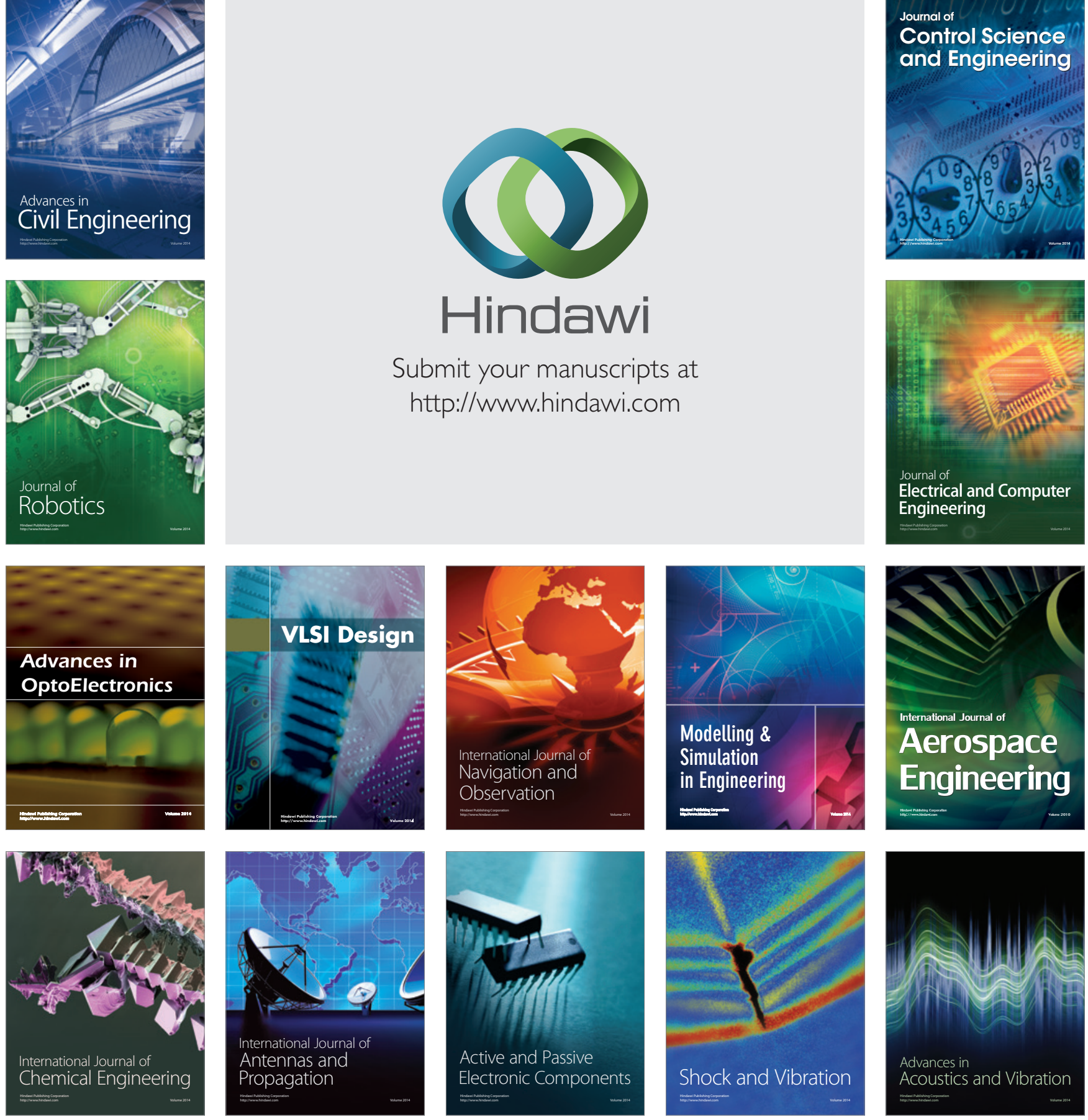